\title{
Flavor Constraints on New Physics
}

\section{Zoltan Ligeti*}

Lawrence Berkeley National Laboratory, University of California, Berkeley, CA 94720, USA

E-mail: ligeti@berkeley.edu

This talk highlights, from a theoretical point of view, some recent exciting results in flavor physics, as well as future prospects. We discuss possible implications of a subset of the experimental results in tension with the standard model, such as the $4 \sigma$ deviation in the $B \rightarrow D^{(*)} \tau \bar{v}$ decay rates, and recent improvements in the constraints on axion portal dark matter models. We use the examples of constraining new physics contributions to neutral meson mixing and the search for possible vector-like fermions to illustrate the expected progress over the next decade to increase the sensitivity to new physics at shorter distance scales. We also speculate about the ultimate limitations of (quark) flavor physics probes of new physics.

XXVII International Symposium on Lepton Photon Interactions at High Energies

17-22 August 2015

Ljubljana, Slovenia

${ }^{*}$ Speaker. 


\section{Introduction}

I was asked to talk about flavor physics constraints on new physics (NP). A slight complication is that in the absence of unambiguous observations of deviations from the standard model (SM) in laboratory experiments so far, and with the LHC pushing the scale of NP higher, there are not really good "simplified models" for non-SM flavor physics, containing a modest number of parameters, which a large class of NP models match onto. There are many flavor physics constraints, and there are many NP models, and attempts to simplify this large (and for most people uninspiring) matrix of constraints has only achieved limited success for a handful of processes. In other words, the interesting information from flavor physics is not simple (as it depends on a large number of processes and the theory is often complicated), and the simple information from flavor physics is not interesting; for example, we learn little from just the values of Cabibbo-Kobayashi-Maskawa $(\mathrm{CKM})$ matrix elements ${ }^{1}$.

Flavor physics is the study of interactions that distinguish the three generations of fermions, i.e., interactions that break the global $[U(3)]^{5}$ symmetry of the SM, of which each $U(3)$ acts on one of the 5 fermion representations $(Q, u d, L, e)$. In the SM, this symmetry is broken by the Yukawa couplings, while in the presence of new physics there generally are additional sources of flavor (and $C P$ ) violation. We do not understand the flavor structure of the SM, and if there is new physics at the $1-100 \mathrm{TeV}$ scale, we have to understand the mechanism that suppresses its effects in the flavor sector to satisfy the experimental constraints. In addition, the observed baryon asymmetry of the Universe requires $C P$ violation beyond the SM (although that need not occur in the quark sector, nor necessarily in flavor changing processes). In any case, flavor measurements provide rich and sensitive ways to probe the SM and search for NP, and flavor physics strongly constrains any NP within the LHC reach. These measurements will reach much higher sensitivities in the next decade.

The sensitivity of flavor measurements to very high mass scales typically comes from large SM suppressions. As a simple (and historically important) example, consider $K^{0}-\bar{K}^{0}$ mixing. The splitting between the two mass eigenstates is $\Delta m_{K} / m_{K} \sim 7 \times 10^{-15}$. In the SM, $\Delta m_{K}$ arises dominantly from box diagrams with virtual $W$ bosons and $c$ quarks, and can be estimated as

$$
\frac{\Delta m_{K}}{m_{K}} \sim \alpha_{w}^{2}\left|V_{c s} V_{c d}\right|^{2} \frac{m_{c}^{2}}{m_{W}^{4}} f_{K}^{2} .
$$

The result is suppressed by CKM angles, a loop factor, the weak coupling, and the GIM mechanism. If a heavy particle, $X$, with effective $\bar{s} d X$ coupling, $g$, contributes an $\mathscr{O}(1)$ fraction to $\Delta m_{K}$, then

$$
\left|\frac{\Delta m_{K}^{(X)}}{\Delta m_{K}^{(\exp )}}\right| \sim\left|\frac{g^{2} \Lambda_{\mathrm{QCD}}^{3}}{M_{X}^{2} \Delta m_{K}^{(\exp )}}\right| \Rightarrow \frac{M_{X}}{g} \gtrsim 2 \times 10^{3} \mathrm{TeV} .
$$

So even TeV-scale particles with loop-suppressed couplings $\left[g \sim \mathscr{O}\left(10^{-3}\right)\right]$ can give observable effects. Thus, flavor measurements probe the TeV scale if the NP has SM-like flavor structure, and much higher scales if the NP flavor structure is generic. In the SM only $W$ bosons change fermion flavor, so flavor-changing processes of the known fermions (except the $t$ quark) are suppressed by at least the second power of a high scale $\left(G_{F} \propto 1 / m_{W}^{2}\right.$ in the SM, or $\left.1 / M_{\mathrm{NP}}^{2}\right)$ and often

\footnotetext{
${ }^{1}$ As Lincoln Wolfenstein sometimes said, even though he invented the Wolfenstein parameters, he did not care what their values were, only whether many overconstraining measurements gave consistent determinations.
} 


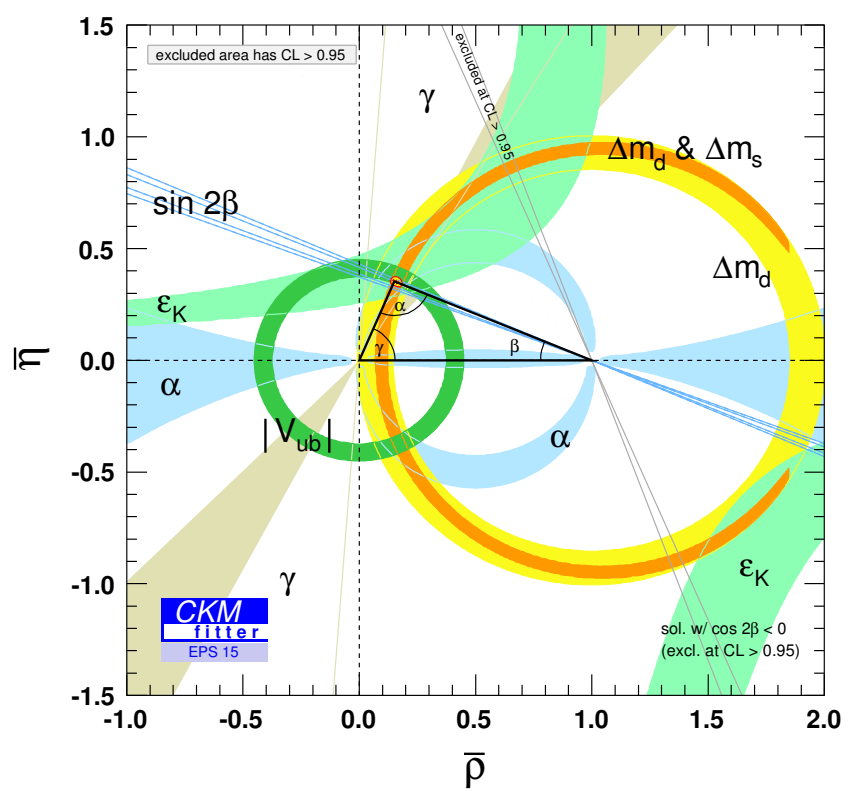

Figure 1: The standard model CKM fit, and individual constraints (colored regions show 95\% CL) [5].

by additional small coefficients. We want to find out if the higher dimension operators generated by the high-scale physics have coefficients as predicted by the SM, and if operators forbidden in the SM (e.g., right handed currents) are generated.

While new physics has been widely expected to occur at the $\mathrm{TeV}$ scale, hinted by the hierarchy problem and the WIMP paradigm, after the discovery of the Higgs boson, no other new particle is guaranteed to be observable in near future laboratory experiments. In flavor physics, typically kaons probe the highest scales, since the SM suppressions are strongest for flavor-changing neutral currents (FCNC) between 1st and 2nd generation quarks. In many NP scenarios the 3rd generation is rather different from the first two, so there is strong motivation to explore what the technology allows us to probe. I find it fairly certain that if new physics is discovered, we will eventually understand it as "natural", no matter how "strange" it might seem at first.

Section 2 summarizes the current status of (quark) flavor physics and reviews some tensions with the SM predictions. These are some of the most often discussed topics recently, and they are also interesting because they may have the best chance to be established as clear deviations from the $\mathrm{SM}$, as more data is accumulated. (I include four recent measurements [1, 2, 3, 4] for completeness, which appeared since the conference.) Section 3 gives some examples of the expected future progress and improvements in sensitivity to NP, independent of the current data. Section 4 contains some comments on the ultimate sensitivity of flavor physics experiments to NP.

\section{Status of flavor physics}

A detailed introduction to flavor physics is omitted here, as well as a review of the determinations of CKM elements; see, e.g., Refs. [6,7]. The magnitudes of CKM elements are mainly extracted from semileptonic and leptonic $K, D$, and $B$ decays, and $B_{d, s}$ mixing. These determine the sides of the unitarity triangle shown in Fig. 1, which is a convenient way to compare many 


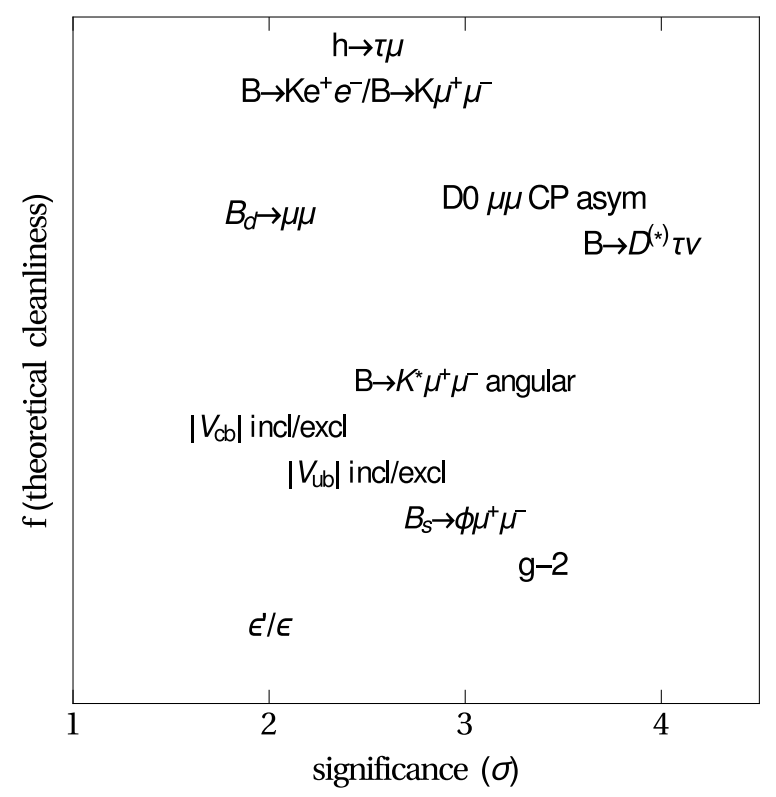

Figure 2: Some recent measurements in tension with the SM. The horizontal axis shows the nominal significance. The vertical axis shows (monotonically, in my opinion) an undefined function of an ill-defined variable: the theoretical cleanliness. That is, the level of plausibility that a really conservative estimate of the theory uncertainty of each observable may affect the significance of its deviation from the SM by $1 \sigma$.

constraints on the SM and visualize the level of consistency. Any constraint which renders the area of the unitarity triangle nonzero, such as nonzero angles $(\bmod \pi)$, has to measure $C P$ violation, and were reviewed in another talk [8]. Some of the most important measurements are shown in Fig. 1, together with the CKM fit in the SM. (The notation $\bar{\rho}, \bar{\eta}$ instead of $\rho, \eta$ simply corresponds to a small modification of the original Wolfenstein parametrization, to keep unitarity exact.) While Fig. 1 shows very good consistency, it does not address how large new physics contributions are allowed. As we see below, in the presence of new physics the fit becomes less constrained, and $\mathscr{O}(20 \%)$ NP contributions to most FCNC processes, relative to the SM, are still allowed.

Several measurements show intriguing deviations from the SM predictions. Some of those that reach the $2-4 \sigma$ level are depicted schematically in Fig. 2. The horizontal axis shows the nominal significance and the vertical axis relates to the theoretical cleanliness of the SM predictions. What I mean is some (monotonic) measure of the plausibility that a conservative estimate of the theory uncertainty may affect the overall significance by $1 \sigma$. All of these are frequently discussed, some have triggered hundreds of papers, and could be the subjects of entire talks each.

Currently, the $B \rightarrow D^{(*)} \tau \bar{v}$ rates, specifically the $R\left(D^{(*)}\right)=\Gamma\left(B \rightarrow D^{(*)} \tau \bar{\nu}\right) / \Gamma\left(B \rightarrow D^{(*)} l \bar{v}\right)$ ratios (where $l=e, \mu$ ) constitute the most significant discrepancy from the SM in collider experiments $[9,10,11,12,1]$ (aside from neutrino masses). The effect is at the $4 \sigma$ level [13]. Figure 3 shows the current data, the SM expectations, as well as the expected Belle II sensitivity. These measurements show good consistency with one another. The theory is also on solid footing, since heavy quark symmetry suppresses model independently the hadronic physics needed for the SM prediction, most of which is actually constrained by the measured $B \rightarrow D^{(*)} l \bar{v}$ decay distributions. 


\begin{tabular}{c|cc}
\hline \hline & $R(D)$ & $R\left(D^{*}\right)$ \\
\hline BaBar & $0.440 \pm 0.058 \pm 0.042$ & $0.332 \pm 0.024 \pm 0.018$ \\
Belle & $0.375 \pm 0.064 \pm 0.026$ & $0.293 \pm 0.038 \pm 0.015$ \\
Belle & & $0.302 \pm 0.030 \pm 0.011$ \\
LHCb & & $0.336 \pm 0.027 \pm 0.030$ \\
\hline Exp. average & $0.397 \pm 0.040 \pm 0.028$ & $0.316 \pm 0.016 \pm 0.010$ \\
\hline my SM expectation & $0.300 \pm 0.010$ & $0.252 \pm 0.005$ \\
\hline Belle II, 50/ab & \pm 0.010 & \pm 0.005 \\
\hline \hline
\end{tabular}

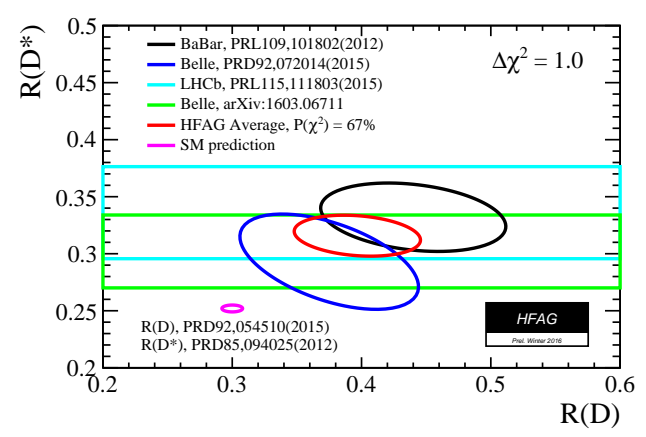

Figure 3: Left: measurements of $R\left(D^{(*)}\right)$ [9, 11, 12, 1], their averages [13], the SM predictions [14, 15, 16], and future sensitivity [17]. Right: the measurements, world average (red), and SM prediction (magenta).

It is somewhat surprising to find such large deviations from the SM in processes which occur at tree level in the SM. The central values of the current world averages would imply that there has to be new physics at a fairly low scale. Some scenarios are excluded by LHC Run 1 bounds already, and many more will soon be constrained by LHC Run 2 data. To fit the current central values, mediators with leptoquark or $W^{\prime}$ quantum numbers are preferred compared to scalars. Leptoquarks are favored if one requires the NP to be minimally flavor violating (MFV), which helps explain the absence of other flavor signals and suppress direct production of the new particles at the LHC from partons abundant in protons [18]. There are several options for the lepton-flavor structure of the new physics, which can have "lepton-MFV" or " $\tau$-alignment" [18]. For example, the latter can be realized with an $A_{4}$-type symmetry for the leptons, which links it to neutrino flavor [19]. To illustrate the wide range of possibilities, there are viable scenarios in which $B \rightarrow D^{(*)} \tau \bar{v}$ are SM-like, but $B \rightarrow D^{(*)} l \bar{v}$ are suppressed by interference between NP and the SM [20].

There are many further experimental measurements that can be done to clarify this anomaly. The $B \rightarrow D^{(*)} \tau \bar{v}$ rates seem to exceed [18] the LEP measurements of the inclusive $b \rightarrow X \tau \bar{\nu}$ rate [7], and the inclusive $B \rightarrow X_{c} \tau \nu$ rate [21] has not yet been measured. The equality of the $e$ and $\mu$ rates are not well constrained, and the currently allowed differences [22, 23] open up (or keep open) model building options [24]. In many scenarios, bounds on $b \rightarrow s v \bar{v}$ processes are very important $[18,25]$. A lot will be learned, hopefully soon, from LHCb result on $R(D)$, measurements using hadronic $\tau$ decays, measurements in $\Lambda_{b}$ and $B_{s}$ decays, and later from Belle II. If a deviation from the SM is established, it will strongly motivate to measure all possible semitauonic modes, both in $b \rightarrow c$ and $b \rightarrow u$ transitions [26, 27].

Another measurement which has drawn immense attention is the " $P_{5}^{\prime}$ anomaly" in a $B \rightarrow$ $K^{*} \mu^{+} \mu^{-}$angular distribution (see, e.g., Refs. [28, 29]), measured at LHCb [30] and recently at Belle [2], and discussed in another talk in more detail [31]. The measurements are shown in the left plot in Fig. 4, together with a SM prediction [32]. These "optimized observables" are based on the SCET factorization theorem for semileptonic $B$ decay form factors [33, 34], and constructing combinations from which the "nonfactorizable" ("soft") contributions cancel. (These are nonperturbative functions of $q^{2}$, which obey symmetry relations [35]; additional terms are either power suppressed or contain an explicit $\alpha_{s}$ factor.) The magnitudes of the correction terms, that is one's ability to calculate the form factor ratios at small $q^{2}$ reliably, is debated [36] (and are not well constrained by data yet). The tension between theory and the data is certainly intriguing, and many 

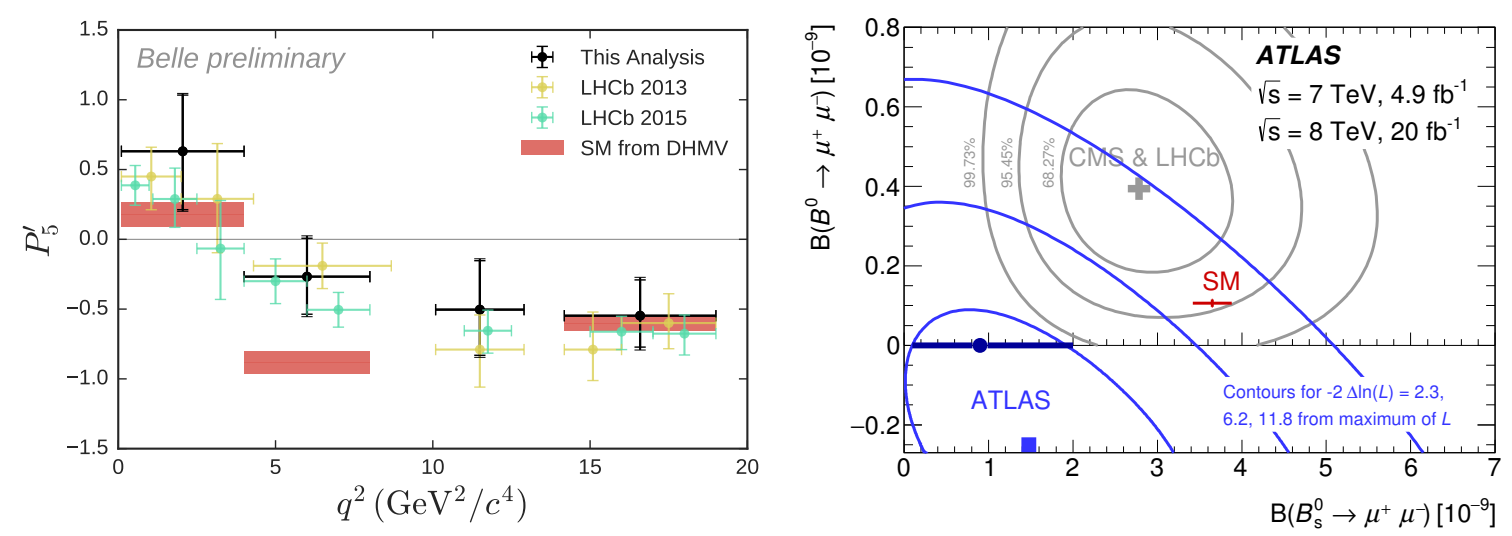

Figure 4: Left: The LHCb [30] and Belle [2] measurements of $P_{5}^{\prime}$ in $B \rightarrow K^{*} \mu^{+} \mu^{-}$. Right: The $B_{s, d} \rightarrow$ $\mu^{+} \mu^{-}$measurements from LHCb and CMS [44], and the ATLAS constraints [3] superimposed.

studies exist both in terms of model independent fits and specific model predictions. Some of the simplest models are $Z^{\prime}$-like, with nonuniversal flavor couplings. One may be concerned that the best fit is a new contribution to the operator $O_{9}=e^{2}\left(\bar{s} \gamma_{\mu} P_{L} b\right)\left(\bar{\ell} \gamma^{\mu} \ell\right)$ in the effective Hamiltonian, the same term which would be modified if theoretical control over the $c \bar{c}$ loop contributions were worse than expected. (This was also emphasized recently in Ref. [37].) There are many possible connections to the $\sim 2.5 \sigma$ anomaly in $\Gamma\left(B \rightarrow K e^{+} e^{-}\right) \neq \Gamma\left(B \rightarrow K \mu^{+} \mu^{-}\right)$as well [38].

For these observables, too, I trust that with improved measurements and theory, the source of the currently seen effects will be understood. With more data, one can test the $q^{2}$ (in)dependence of the extracted Wilson coefficients. In the large $q^{2}$ (small recoil) region one can make model independent predictions both for exclusive [39] inclusive [40] $b \rightarrow s l^{+} l^{-}$mediated decays, which is complementary to the small $q^{2}$ region, and has different theory uncertainties.

Another anomaly observed is the $B_{s} \rightarrow \phi \mu^{+} \mu^{-}$rate in the $1<q^{2}<6 \mathrm{GeV}^{2}$ region being about $3 \sigma$ below theoretical calculations [41]. This relies on QCD sum rules [42] combined with lattice QCD calculations of the form factors at large $q^{2}$ [43]. Extending the lattice results to lower $q^{2}$ would help clarify the picture, as well as more precise measurements, also at high $q^{2}$.

If new physics is at play in these processes, it is likely to impact $B \rightarrow \mu^{+} \mu^{-}$, too. The combined LHCb and CMS observation [44] of $B_{s} \rightarrow \mu^{+} \mu^{-}$, the constraint on $B_{d} \rightarrow \mu^{+} \mu^{-}$, and the recent ATLAS [3] constraints are shown in the right plot in Fig. 4, as well as the SM prediction. Measuring a rate at the $3 \times 10^{-9}$ level is impressive, and future refinements are high priority. The nonperturbative input in this case is just $f_{B}$, which is under good control in lattice QCD.

Another deviation from the SM expectations, which is theoretically very clean, and has been $3-4 \sigma$, is the $\mathrm{D} \emptyset$ measurement of the like-sign dimuon charge asymmetry in semileptonic decays of $b$ hadrons, $\left(N_{\mu^{+} \mu^{+}}-N_{\mu^{-} \mu^{-}}\right) /\left(N_{\mu^{+} \mu^{+}}+N_{\mu^{-} \mu^{-}}\right)$[45], shown in the left plot in Fig. 5. A nonzero signal could come from a linear combination of $C P$ violation in $B_{s}$ and $B_{d}$ mixing, $a_{\mathrm{SL}}^{d, s}$ (see, e.g., Ref. [46]), and the SM prediction is well below the current sensitivity. Separate measurements of $a_{\mathrm{SL}}^{d}$ and $a_{\mathrm{SL}}^{s}$ from BaBar, Belle, DØ, and LHCb are consistent in with the SM. The very recent LHCb measurement of $a_{\mathrm{SL}}^{s}=(0.39 \pm 0.33) \%$ with $3 / \mathrm{fb}$ [4], reducing the uncertainty from $0.62 \%$ with $1 / \mathrm{fb}$, starts to be in tension with the $\mathrm{D} \varnothing$ anomaly. If there is new physics in $C P$ violation in $B_{s}$ mixing, then one may also expect to see a deviation from the SM in the time-dependent $C P$ asym- 

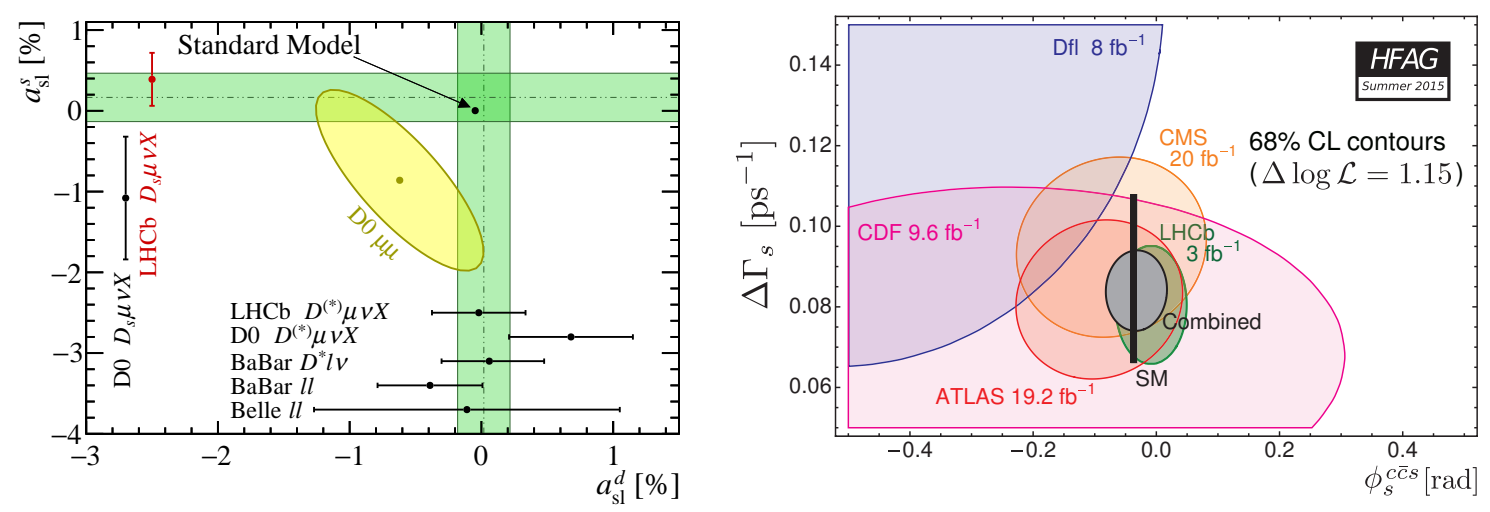

Figure 5: Left: bounds on $C P$ violation in $B_{d, s}$ mixing, $a_{\mathrm{SL}}^{d, s}$ [4]. The vertical and horizontal bands show the averages of the separate $B_{d}$ and $B_{s}$ measurements, respectively, and the yellow ellipse is the DØ measurement. Right: measurements of $\phi_{s} \equiv-2 \beta_{s}$ showing good consistency with the SM.

metry in $B_{s} \rightarrow J / \psi \phi$. Recent LHC measurements, however, are consistent with the SM, as shown in the right plot in Fig. 5. Most importantly, the theory uncertainties are well below the experimental sensitivity in the coming years, so a lot can be learned from more precise measurements.

Understanding the long-standing tensions between inclusive and exclusive measurements of $\left|V_{c b}\right|$ and $\left|V_{u b}\right|$ are important for NP searches. The ratio $\left|V_{u b} / V_{c b}\right|$ together with $\gamma$ determine the apex of the unitarity triangle from tree-level processes, which is crucial for improving the sensitivity to $\mathrm{NP}$ in $B$ mixing and in $C P$ violation measurements involving loop processes. Understanding the QCD dynamics of semileptonic $B$ decays is also important, because the theoretical tools coincide with those used in inclusive and exclusive $b \rightarrow s \gamma$ and $b \rightarrow s \ell \bar{\ell}$ decays. While I have also entertained modifications of these CKM measurements due to NP (such as right-handed currents [47]), many known theoretical and experimental improvements can take place in the future, such as doing all measurements in events where the other $B$ is fully reconstructed.

The muon $g-2$ measurement has a more than $3 \sigma$ tension with the SM, according to most analysis. It may be a sign of new physics, but some complicated strong interaction dynamics could still be at play and decrease the significance of the deviation. One hopes that lattice QCD will determine reliably the hadronic light-by-light scattering and vacuum polarization contributions. While supersymmetric models with relatively light sleptons can still account for the deviation [48], the LHC will improve limits on such NP explanations, and sub-GeV weakly coupled new particles could also be at play [49]. For the heavy NP explanation, somewhat puzzling is that the required new physics contribution is the same size as the one-loop SM electroweak contribution.

It has long been known that kaon $C P$ violation is sensitive to some of the highest energy scales. For the $\varepsilon$ parameter, the SM is in good agreement with the data, and the NP contribution is constrained to be $\lesssim 30 \%$ of that of the SM [50]. Calculating the SM prediction for direct $C P$ violation, the $\varepsilon^{\prime}$ parameter, has been a multi-decade challenge, and important progress has been made recently [51]. Results with several lattice spacings are needed to decide if NP is present.

These experimental hints of possible deviations from the SM are fantastic for several reasons. Unambiguous evidence for NP would obviously be the start of a new era, and one would also get a rough upper bound on the scale of NP, even if it is not seen directly at ATLAS \& CMS. It is also 

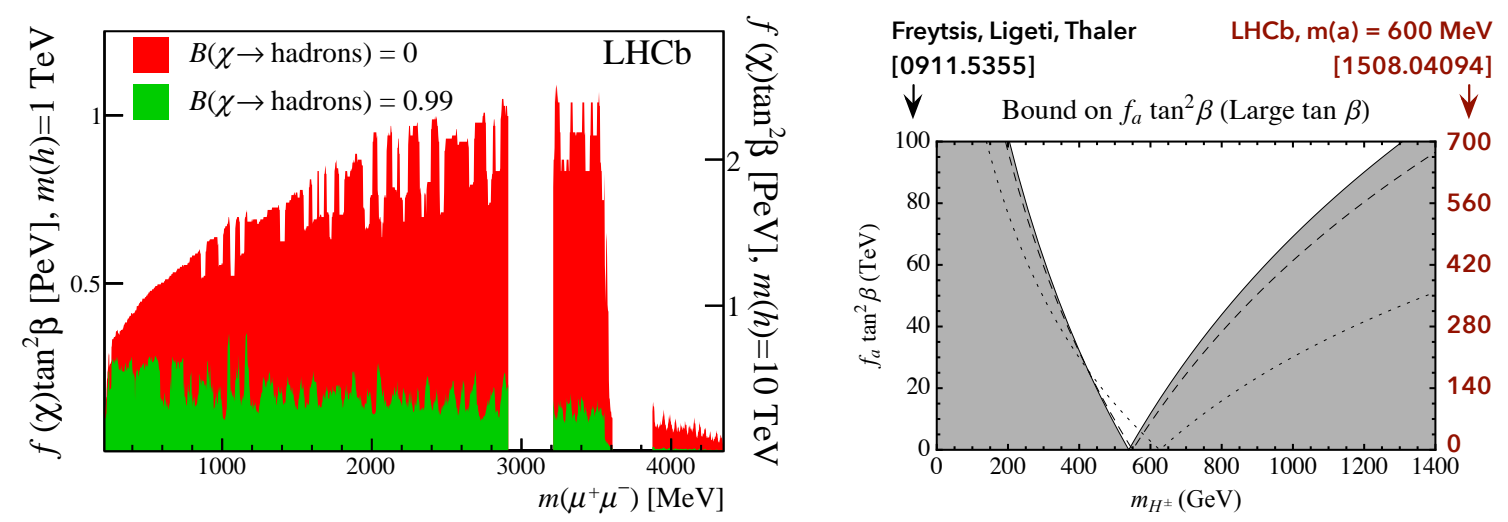

Figure 6: Left: LHCb bounds on $f_{\chi}^{2} \tan ^{2} \beta$ as a function of $m_{\mu+\mu^{-}}$[57] in the model [59]. Right: The bound as a function of $m_{H^{ \pm}}$in the same model; the right axis shows a nearly order of magnitude improvement.

useful to have experimental results challenge theory, since unexpected signals motivate both model building and revisiting the SM predictions. This was the case with the Tevatron anomaly in the $t \bar{t}$ forward-backward asymmetry, $A_{\mathrm{FB}}^{t \bar{\tau}}$, which disappeared due to refinements of the experimental results (the SM predictions also improved [52]). Concerning the recent $3 \sigma$ hint for direct $C P$ violation in the difference of $C P$ asymmetries in $D \rightarrow K^{+} K^{-}$and $D \rightarrow \pi^{+} \pi^{-}, \Delta A_{C P}=A_{K^{+} K^{-}}-$ $A_{\pi^{+} \pi^{-}}$, I doubt the initial measurement near $1 \%$ could be attributed to the SM [53]. The central value of the world average has decreased since 2012, as has the significance of the hint for $\Delta A_{C P} \neq$ 0 . We probably still do not know how large $\Delta A_{C P}$ the SM could generate. However, exploring it taught us, for example, about how much (or how little) the quark and squark mixing matrices can differ and squark masses (don't) need to be degenerate [54, 55] in alignment models [56].

A recent measurement in which no anomaly is seen, but I find the nearly order of magnitude increase in mass-scale sensitivity due to a recent LHCb analysis [57] impressive, is the search for an axion-like particle, coupling to SM fermions as $\left(m_{\psi} / f_{a}\right) \bar{\psi} \gamma_{5} \psi a$, explained in another talk [31]. Such models are also interesting, because they may have highly suppressed spin-independent direct detection cross sections [58]. The left plot in Fig. 6 shows the 95\% CL lower bound on $f_{\chi}^{2} \tan ^{2} \beta$ in the model of Ref. [59], from the absence of a narrow $\mu^{+} \mu^{-}$peak in $B \rightarrow K^{*} \chi\left(\chi \rightarrow \mu^{+} \mu^{-}\right)$as a function of $m_{\mu^{+}} \mu^{-}$. The bound is shown for $m_{H^{ \pm}}=1 \mathrm{TeV}$ and two values of the hadronic branching fraction of the axion-like particle. The right plot shows the bound on the same quantity as a function of $m_{H^{ \pm}}\left(f_{a}\right.$ in [59] is $f_{\chi}$ in [57]). The left vertical axis is the bound estimated in 2009 [59] from BaBar \& Belle data with only a few bins, and the right vertical axis shows the LHCb bound [57]. The dashed (dotted) curve shows the bound for $\tan \beta=3(\tan \beta=1)$. In this model, for any value of $\tan \beta$, the NP contribution vanishes due to a cancellation for a certain value of $m_{H^{ \pm}}$.

\section{Future increases in new physics scales probed}

I would like to talk about three topics briefly in this part: (i) the future theory uncertainty of the measurement on $\sin 2 \beta$ from $B \rightarrow J / \psi K_{S}$; (ii) the future sensitivity to NP in mixing of neutral mesons; (iii) sensitivity of flavor physics experiments to very heavy vector-like fermions. 


\subsection{What are the ultimate theory uncertainties of $\sin 2 \beta$ ?}

The theoretical uncertainty of the SM predictions for the time dependent $C P$ asymmetries in the "gold-plated" modes $B \rightarrow J / \psi K_{S}$ and $B_{s} \rightarrow J / \psi \phi$ are of great importance. They arise from contributions to the decay amplitude proportional to $V_{u b} V_{u s}^{*}$ instead of the dominant $V_{c b} V_{c s}^{*}$ terms. I refer to this as $V_{u b}$ contamination, instead of the often used penguin pollution phrase (which is less correct and less clear). This effect did not matter in practice in the past, but it will be important for interpreting the full $\mathrm{LHCb}$ and Belle II data sets. During the BaBar/Belle era, the experimental precision was an order of magnitude above the nominal magnitude of the theoretical uncertainty, $\lambda^{2}\left(\alpha_{s} / \pi\right) \sim 0.004$. So even a factor of few enhancement of the latter did not matter. ${ }^{2}$ A number of approaches have been developed, using a combination of diagrammatic and flavor symmetry arguments with various assumptions [62,63]. (I hasten to add a triviality: there is no relation based only on $S U$ (3) flavor symmetry between final states which are entirely in different representations; e.g., $\phi$ is an $S U(3)$ singlet and $\rho \& K^{*}$ are members of an octet.) The experimental tests performed so far [8] do not indicate big enhancements of the theory uncertainties.

The question that really matters in my opinion is not what it takes to set plausible upper bounds on the $V_{u b}$ contamination, while the measurements agree with the SM, but what it would take to convince the community that NP is observed at LHCb and Belle II, especially if no NP is seen by ATLAS and CMS. Therefore, one cannot overemphasize the importance of starting from rigorous theoretical foundations, with well defined expansion parameter(s).

A relation based only on $S U$ (3) flavor symmetry, which cancels the $V_{u b}$ contamination in $\sin 2 \beta$ against other observables in the $S U(3)$ limit, was constructed recently [64]

$$
\sin 2 \beta=\frac{S_{K_{S}}-\lambda^{2} S_{\pi^{0}}-2\left(\Delta_{K}+\lambda^{2} \Delta_{\pi}\right) \tan \gamma \cos 2 \beta}{1+\lambda^{2}} .
$$

Here $S_{h}(h=K, \pi)$ is the usual coefficient of the $\sin (\Delta m t)$ term in the time-dependent $C P$ asymmetry [7] in $B \rightarrow J / \psi h^{0}, \lambda \simeq 0.225$ is the Wolfenstein parameter,

$$
\Delta_{h}=\frac{\bar{\Gamma}\left(B_{d} \rightarrow J / \psi h^{0}\right)-\bar{\Gamma}\left(B^{+} \rightarrow J / \psi h^{+}\right)}{\bar{\Gamma}\left(B_{d} \rightarrow J / \psi h^{0}\right)+\bar{\Gamma}\left(B^{+} \rightarrow J / \psi h^{+}\right)},
$$

and $\bar{\Gamma}$ denotes the $C P$ averaged rates. Using Eq. (3.1) it is possible to replace the $V_{u b}$ contamination in the $\sin 2 \beta \simeq S_{K_{S}}$ relation with isospin breaking, which could be smaller than the possibly enhanced $V_{u b}$ contamination one wants to constrain. It also provides redundancy, replacing one theory uncertainty with a different one. For the $V_{c b} V_{c s}^{*}$ terms in the effective Hamiltonian, $\Delta_{K, \pi}$ violate isospin, but the $V_{u b} V_{u s}^{*}$ terms generate nonzero $\Delta_{h}$ even in the isospin limit. The resulting constraint on the $\bar{\rho}-\bar{\eta}$ plane is shown in Fig. 7 [40].

Measuring all terms in Eq. (3.1) is not straightforward. Many of the current measurements of $\Delta_{h}$ and the production asymmetry of $B^{+} B^{-}$vs. $B^{0} \bar{B}^{0}$ in $\Upsilon(4 S)$ decay, $f_{+-} / f_{00}$, are circular (the measurements of either assume that the other asymmetry vanishes) [65], so the slight tension in Fig. 7 should be interpreted with caution. To disentangle $\Delta_{h}$ from the production asymmetry,

\footnotetext{
${ }^{2}$ Until 1997 this estimate was often written as $\lambda^{2}\left(\alpha_{S} / 4 \pi\right)$. Omitting the factor 4 anticipates some enhancement of the penguin matrix element, observed in charmless $B$ decays [60] but not yet well constrained in decays to charmonia. Better calculable $\mathscr{O}\left(10^{-3}\right)$ effects arise from $C P$ violation in $K$ and $B$ mixing, and the $\Gamma_{B_{L}}-\Gamma_{B_{H}}$ width difference [61].
} 


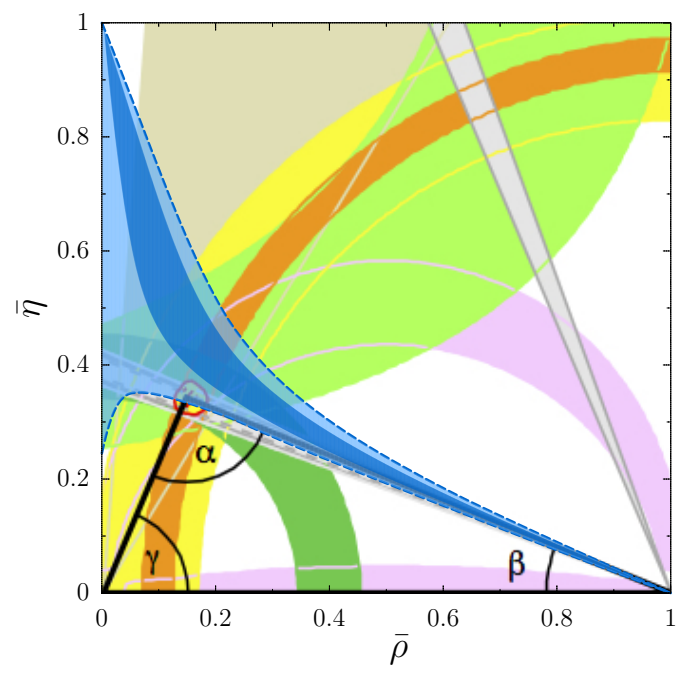

Figure 7: The dark (light) blue region shows the $1 \sigma(2 \sigma)$ constraint in the $\bar{\rho}-\bar{\eta}$ plane from Eq. (3.1) [64].

more precise measurements of the latter are needed. One option may be to utilize that isospin violation in inclusive semileptonic decay is suppressed by $\Lambda_{\mathrm{QCD}}^{2} / m_{b}^{2}$ [65]. (Similar suppression of $S U$ (3) symmetry breaking in inclusive $B$ decays by $\Lambda_{\mathrm{QCD}}^{2} / m_{b}^{2}$ is the basis for a theoretically clean prediction for the ratio $\Gamma\left(B \rightarrow X_{s} \ell^{+} \ell^{-}\right) / \Gamma\left(B \rightarrow X_{u} \ell \bar{v}\right)$ at large $q^{2}$ [40]. $)$

It is an open question how well it will be possible to ultimately constrain (convincingly) the size of $V_{u b}$ contamination in the measurements of $\sin 2 \beta$ and $2 \beta_{s}\left(\equiv-\phi_{s}\right)$. Given that $S U(3)$ flavor symmetry has been used to analyze $B$ decays for decades, and previously unknown $S U(3)$ relations can be discovered in 2015 , makes me optimistic that a lot more progress can be achieved.

\subsection{New physics in SM loop processes}

Although the SM CKM fit in Fig. 1 shows impressive and nontrivial consistency, the implications of the level of agreement are often overstated. Allowing new physics contributions, there are a larger number of parameters related to $C P$ and flavor violation, and the fits become less constraining. This is shown in Fig. 8, which shows the determination of the unitarity triangle from tree-dominated decays only, which are unlikely to be affected by new physics. The plot on the left shows the current fit results, while the constraints in the plot on the right is expected to be achievable with $50 \mathrm{ab}^{-1}$ Belle II and $50 \mathrm{fb}^{-1}$ LHCb data [66]. The allowed region in the left plot is indeed significantly larger than in Fig. 1.

It has been known for decades that the mixing of neutral mesons is particularly sensitive to new physics, and probe some of the highest scales. In a large class of models, NP has a negligible impact on tree-level SM transitions (e.g., the measurements of $\gamma,\left|V_{u b}\right|$, and $\left|V_{c b}\right|$ ), and the $3 \times 3$ CKM matrix remains unitary. As a simple example, consider possible NP contributions to $B$ and $B_{s}$ meson mixing, which can be parametrized as

$$
M_{12}=M_{12}^{\mathrm{SM}}\left(1+h_{q} e^{2 i \sigma_{q}}\right), \quad q=d, s .
$$

The constraints on $h_{d}$ and $\sigma_{d}$ in the $B_{d}$ mixing are shown in Fig. 9, and the constraint in the $h_{d}-h_{s}$ plane is shown in Fig. 10. Both plots show the current constraints (left) and those expected to be 

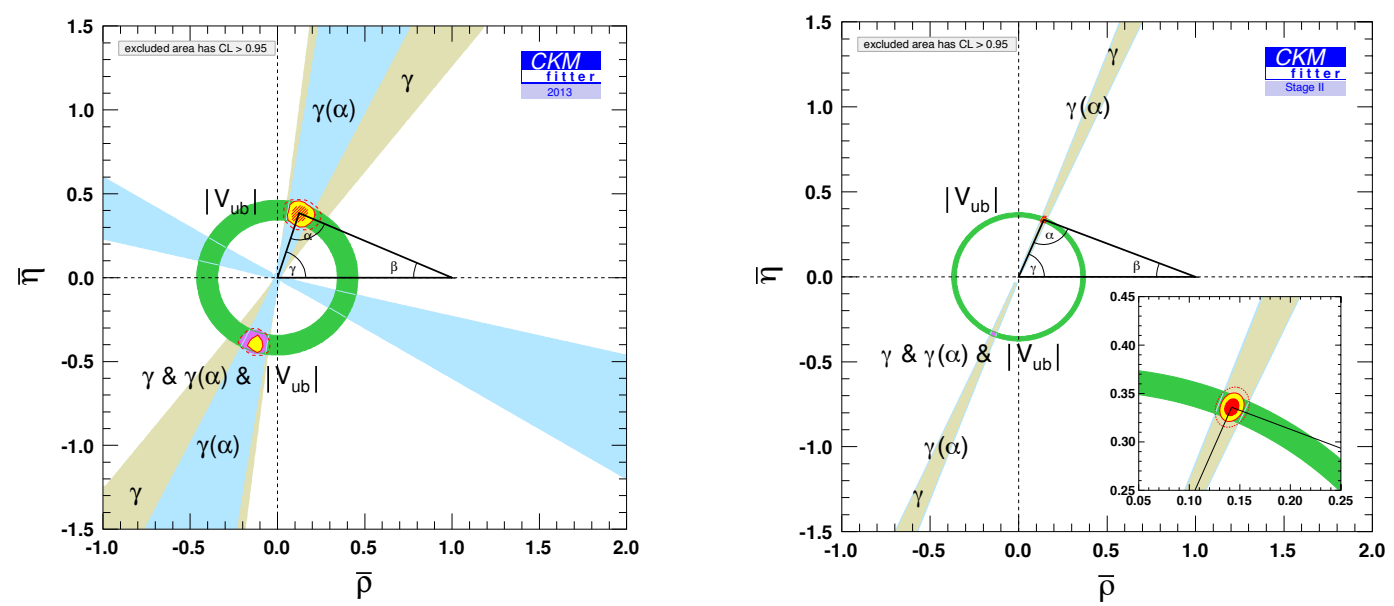

Figure 8: Constraints on $\bar{\rho}-\bar{\eta}$, allowing NP in the $B_{d, s}$ mixing amplitudes (left) and the expectation using $50 \mathrm{ab}^{-1}$ Belle II and $50 \mathrm{fb}^{-1}$ LHCb data (right) [66]. Colored regions show 95\% CL, as in Fig. 1.
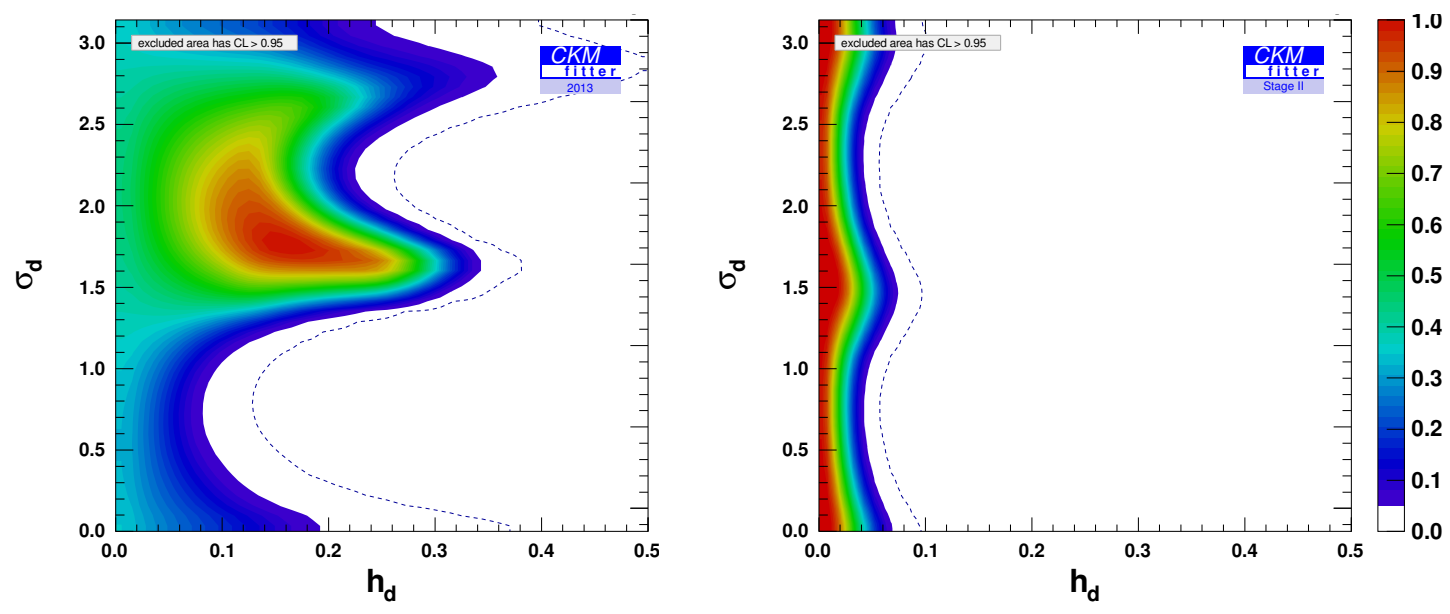

Figure 9: Constraints on the $h_{d}-\sigma_{d}$ parameters (left) and those estimated to be achievable using $50 \mathrm{ab}^{-1}$ Belle II and $50 \mathrm{fb}^{-1}$ LHCb data (right) [66]. Colored regions show $2 \sigma$ limits with the colors indicating CL as shown, while the dashed curves show $3 \sigma$ limits.

achievable with $50 \mathrm{ab}^{-1}$ Belle II and $50 \mathrm{fb}^{-1}$ LHCb data (right) [66]. Figure 9 shows that in the future the bounds on the "MFV-like regions", where NP flavor is aligned with the SM $\left(2 \theta_{d} \simeq 0\right.$ $\bmod \pi$ ), will be comparable to generic values of the NP phase, unlike in the past. Figure 10 shows that the bounds on NP in $B_{s}$ mixing, which were significantly weaker than those in the $B_{d}$ sector until recent $\mathrm{LHCb}$ measurements, are now comparable, and will comparably improve in the future.

As an example, if NP modifies the SM operator describing $B_{q}$ mixing by adding to it a term

$$
\frac{C_{q}^{2}}{\Lambda^{2}}\left(\bar{b}_{L} \gamma^{\mu} q_{L}\right)^{2}
$$

then one finds

$$
h_{q} \simeq \frac{\left|C_{q}\right|^{2}}{\left|V_{t b}^{*} V_{t q}\right|^{2}}\left(\frac{4.5 \mathrm{TeV}}{\Lambda}\right)^{2}
$$



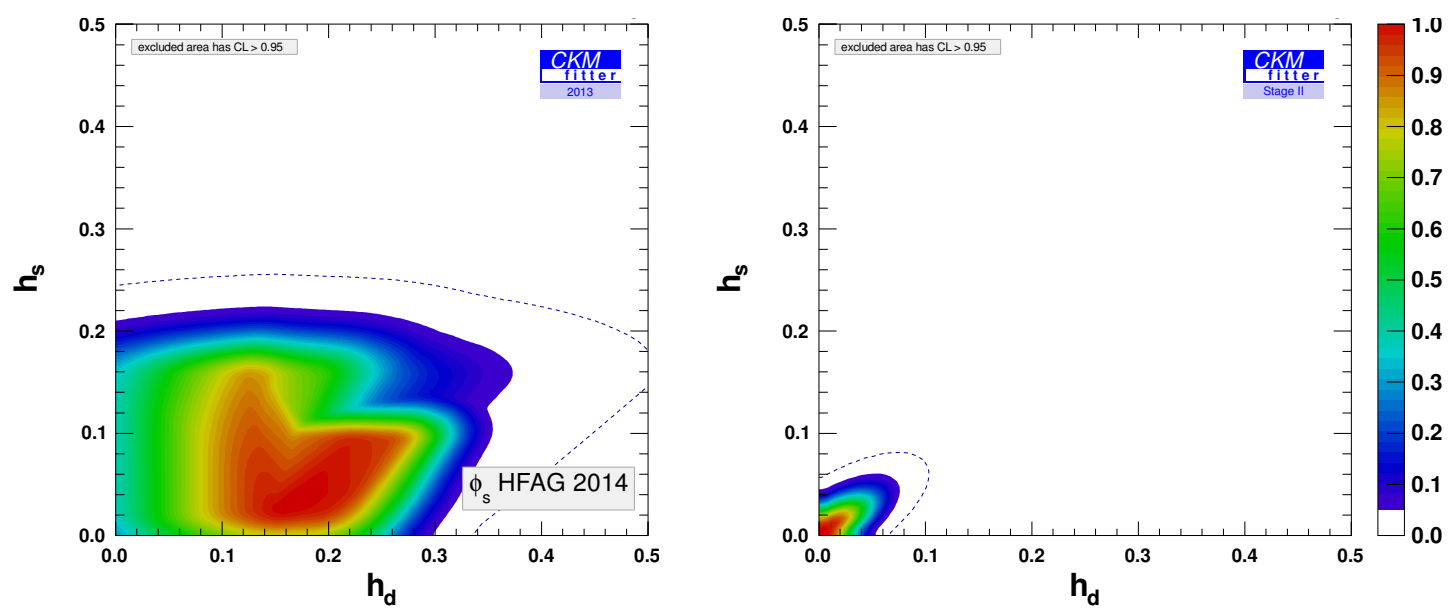

Figure 10: Constraints on the $h_{d}-h_{s}$ parameters now (left plot) and those estimated with $50 \mathrm{ab}^{-1}$ Belle II and $50 \mathrm{fb}^{-1}$ LHCb data (right plot) [66]. The notation is the same as in Fig. 9.

\begin{tabular}{c|c|c|c}
\hline \hline \multirow{2}{*}{ Couplings } & NP loop & \multicolumn{2}{|c}{ Scales (TeV) probed by } \\
& order & $B_{d}$ mixing & $B_{s}$ mixing \\
\hline$\left|C_{q}\right|=\left|V_{t b} V_{t q}^{*}\right|$ & tree level & 17 & 19 \\
$(\mathrm{CKM}-\mathrm{like})$ & one loop & 1.4 & 1.5 \\
\hline$\left|C_{q}\right|=1$ & tree level & $2 \times 10^{3}$ & $5 \times 10^{2}$ \\
(no hierarchy) & one loop & $2 \times 10^{2}$ & 40 \\
\hline \hline
\end{tabular}

Table 1: The scale of the $B_{d, s}$ mixing operators in Eq. (3.4) probed, with $50 \mathrm{ab}^{-1}$ Belle II and $50 \mathrm{fb}^{-1} \mathrm{LHCb}$ data [66]. The differences due to CKM-like hierarchy of couplings and/or loop suppression is shown.

We can then translate the plotted bounds to the scale of new physics probed. The summary of expected sensitivities are shown in Table 1. The sensitivities even with SM-like loop- and CKMsuppressed coefficients are comparable to the scales probed by the LHC in the next decade.

In $K^{0}-\bar{K}^{0}$ mixing the simplest analog of Eq. (3.3) is to parameterize NP via an additive term to the so-called $t t$ contribution in the SM, $M_{12}^{K, t t}=M_{12}^{K, t t}\left(1+h_{K} e^{2 i \sigma_{K}}\right)$. The reason is the short distance nature of NP and the fact that in many NP models the largest contribution to $M_{12}^{K}$ arise via effects involving the third generation. Substantial progress would require lattice QCD to constrain the long distance contribution to $M_{12}^{K}$ at the percent level [66].

There are also strong constraints on NP from $D^{0}-\bar{D}^{0}$ mixing. Since the observed mixing parameters are probably dominated by long distance physics [67], it is hard to improve the bound from simply demanding the NP contribution to be below the measured values of the mixing parameters.

\subsection{Sensitivity to vector-like fermions}

Another illustration of the expected progress with well quantifiable increases in mass scale sensitivity, in both quark and lepton flavor experiments, is to consider extensions of the SM involving vector-like fermions, which can Yukawa couple to the SM [68]. These fermions can have masses $M$ much greater than the weak scale, since they have a mass term even in the absence of 

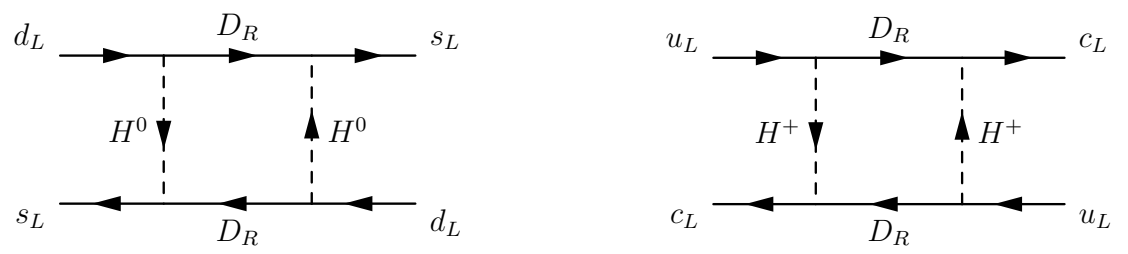

Figure 11: One-loop vector-like fermion contributions to $K$ and $D$ mixing in Model V [68].

electroweak symmetry breaking. These models are a class of simple extensions of the SM, which do not worsen the hierarchy puzzle. There are 11 renormalizable models [68] which add to the SM vector-like fermions in a single (complex) representation of the gauge group that can Yukawa couple to the SM fermions through the Higgs field (4 to leptons, 7 to quarks).

The precise definitions of the $\lambda_{i}$ Yukawa couplings depend on the models, as do the forms of the Lagrangians. For example, what was labeled Model V in Ref. [68] contains vector-like fermions, $D$, with the same quantum numbers as the SM right-handed down-type quarks, which Yukawa couple to the SM left-handed quark doublets $Q_{L}^{i}$ as

$$
\mathscr{L}_{\mathrm{NP}}^{(\mathrm{V})}=\bar{D}(i \not D-M) D-\left(\lambda_{i} \bar{D}_{R} H^{\dagger} Q_{L}^{i}+\text { h.c. }\right),
$$

These new interactions generate $Z$ couplings, e.g., in this Model $V$ to the quarks,

$$
\mathscr{L}_{Z}^{(\mathrm{V})}=-\sum_{i, j}\left(\frac{\lambda_{i}^{*} \lambda_{j} m_{Z}^{2}}{g_{Z} M^{2}}\right) \vec{d}_{L}^{i} \gamma^{\mu} d_{L}^{j} Z_{\mu},
$$

which contribute to, and are constrained by, flavor-changing neutral currents.

These models also generate dimension- 6 four-fermion operators, which contribute to neutral meson mixing. At tree level, the $Z$ contribution in Eq. (3.7) yields coefficients of the form $\left(\lambda_{i} \lambda_{j}^{*}\right)^{2} v^{2} / M^{4}$. At one loop, coefficients of order $\left(\lambda_{i} \lambda_{j}^{*}\right)^{2} /(4 \pi M)^{2}$ are generated, which are neither CKM nor quark-mass suppressed, seemingly not considered in the literature. For large $M$, these one-loop contributions are more important than tree-level $Z$ exchange. They are independent of the Higgs vacuum expectation value, $v$, and arise from short distances $\sim 1 / M$. They can be calculated in the symmetric phase from the box diagrams in Fig. 11 with virtual scalars and the heavy vector-like fermions. The resulting effective Lagrangian in Model V is [68],

$$
\mathscr{L}_{\text {mix }}^{(\mathrm{V})}=-\frac{\left(\lambda_{i}^{*} \lambda_{j}\right)^{2}}{128 \pi^{2} M^{2}}\left[\sum_{k l m n}\left(\bar{u}_{L}^{k} V_{k i} \gamma_{\mu} V_{j l}^{\dagger} u_{L}^{l}\right)\left(\bar{u}_{L}^{m} V_{m i} \gamma_{\mu} V_{j n}^{\dagger} u_{L}^{n}\right)+\left(\bar{d}_{L}^{i} \gamma_{\mu} d_{L}^{j}\right)\left(\bar{d}_{L}^{i} \gamma^{\mu} d_{L}^{j}\right)\right]+\text { h.c. }
$$

Table 2 summarizes the bounds on 5 of the 11 models for illustration. The upper rows for each model show the current bounds, and the lower rows show the expected sensitivities in the next generation of experiments (in the next decade or so). For the vector-like fermions that couple to SM quarks, the bounds are shown separately from $\Delta F=1$ and $\Delta F=2$ processes. For the $\Delta F=2$ bounds on the 1-2 generation couplings, the bounds are shown separately on the real and imaginary parts, since $\varepsilon_{K}$ probes much higher scales than $\Delta m_{K}$ in these models. (In the other cases the differences are of order unity.) We learn that the next generation of experiments will improve the mass scale sensitivities in the leptonic (hadronic) models by up to a factor of $\sim 7(\sim 4)$. 


\begin{tabular}{|c|c|c|c|c|c|c|c|}
\hline \multirow{2}{*}{ Model } & \multirow{2}{*}{$\begin{array}{l}\text { Quantum } \\
\text { numbers }\end{array}$} & \multicolumn{6}{|c|}{ Bounds on $M / \mathrm{TeV}$ and $\lambda_{i} \lambda_{j}$ for each $i j$ pair } \\
\hline & & \multicolumn{2}{|c|}{$i j=12$} & \multicolumn{2}{|c|}{$i j=13$} & \multicolumn{2}{|c|}{$i j=23$} \\
\hline \multirow[t]{2}{*}{ II } & $(1,3,-1)$ & \multicolumn{2}{|c|}{$220^{a}$} & \multicolumn{2}{|c|}{$4.9^{b}$} & \multicolumn{2}{|c|}{$5.2^{c}$} \\
\hline & & \multicolumn{2}{|c|}{$1400^{a}$} & \multicolumn{2}{|c|}{$13^{b}$} & \multicolumn{2}{|c|}{$15^{c}$} \\
\hline \multirow[t]{3}{*}{ III } & $(1,2,-1 / 2)$ & \multicolumn{2}{|c|}{$310^{a}$} & \multicolumn{2}{|c|}{$7.0^{b}$} & \multicolumn{2}{|c|}{$7.4^{c}$} \\
\hline & & \multicolumn{2}{|c|}{$2000^{a}$} & \multicolumn{2}{|c|}{$19^{b}$} & \multicolumn{2}{|c|}{$21^{c}$} \\
\hline & & $\Delta F=1$ & $\Delta F=2$ & $\Delta F=1$ & $\Delta F=2$ & $\Delta F=1$ & $\Delta F=2$ \\
\hline \multirow[t]{2}{*}{ V } & $(3,1,-1 / 3)$ & $66^{d}[100]^{e}$ & $\{42,670\}^{f}$ & $30^{g}$ & $25^{h}$ & $21^{i}$ & $6.4^{j}$ \\
\hline & & $280^{d}$ & $\{100,1000\}^{f}$ & $60^{l}$ & $61^{h}$ & $39^{k}$ & $14^{j}$ \\
\hline \multirow[t]{2}{*}{ VII } & $(3,3,-1 / 3)$ & $47^{d}[71]^{e}$ & $\{47,750\}^{f}$ & $21^{g}$ & $28^{h}$ & $15^{i}$ & $7.2^{j}$ \\
\hline & & $200^{d}$ & $\{110,1100\}^{f}$ & $42^{l}$ & $68^{h}$ & $28^{k}$ & $16^{j}$ \\
\hline \multirow[t]{2}{*}{ XI } & $(3,2,-5 / 6)$ & $66^{d}[100]^{e}$ & $\{42,670\}^{f}$ & $30^{g}$ & $25^{h}$ & $18^{k}$ & $6.4^{j}$ \\
\hline & & $280^{d}$ & $\{100,1000\}^{f}$ & $60^{l}$ & $61^{h}$ & $39^{k}$ & $14^{j}$ \\
\hline
\end{tabular}

Table 2: Bounds in some of the vector-like fermion models [68] on $M[\mathrm{TeV}] / \sqrt{\left|\lambda_{i} \lambda_{j}\right|}$ in the leptonic models, and from the $\Delta F=1$ constraints on the hadronic models. The $\Delta F=2$ bounds show $M / \sqrt{\left|\lambda_{i} \lambda_{j}\right|^{2}}$, except for $K$ meson mixing we show $\left\{M / \sqrt{\left|\operatorname{Re}\left(\lambda_{i} \lambda_{j}^{*}\right)^{2}\right|}, M / \sqrt{\left|\operatorname{Im}\left(\lambda_{i} \lambda_{j}^{*}\right)^{2}\right|}\right\}$. The strongest bounds arise, or are expected to arise, from: $a) \mu$ to $e$ conversion; $b) \tau \rightarrow e \pi$; c) $\tau \rightarrow \mu \rho$; $d) K \rightarrow \pi \nu \bar{v}$; $e$ ) $K_{L} \rightarrow \mu^{+} \mu^{-}$(this involves $\left|\operatorname{Re}\left(\lambda_{1} \lambda_{2}^{*}\right)\right|$ and is in square brackets because prospects for improvements are weak); $f$ ) $K$ mixing; g) $B \rightarrow \pi \mu^{+} \mu^{-}$; h) $B_{d}$ mixing; i) $\left.B \rightarrow X_{s} \ell^{+} \ell^{-} ; j\right) B_{s}$ mixing; $\left.\left.k\right) B_{s} \rightarrow \mu^{+} \mu^{-}, l\right) B_{d} \rightarrow \mu^{+} \mu^{-}$.

\subsection{Top, higgs, and new physics flavor}

These are vast topics which I could not cover in detail in the talk, nor is it possible here.

Top quarks in the SM decay almost exclusively to $b W$, with the second largest branching fraction $\mathscr{B}(t \rightarrow s W)<2 \times 10^{-3}$. Particularly clean probes of the SM are FCNC top decays, for which the SM predictions are below the $10^{-12}$ level. The current bounds are roughly at the level $\mathscr{B}(t \rightarrow q Z) \lesssim 10^{-3}, \mathscr{B}(t \rightarrow q g) \lesssim 10^{-4}$, and $\mathscr{B}(t \rightarrow q h) \lesssim 0.5 \%$, with the precise limits depending on the ratio of $q=u, c$ produced by new physics [69]. The ultimate LHC sensitivities are expected to be about a factor of $10^{2}$ better, hence any observation would be a clear sign of NP. There is obvious complementarity between FCNC searches in the top sector, and low energy flavor physics bounds. Since $t_{L}$ is in the same $S U(2)$ doublet as $b_{L}$, several operators have correlated effects in $t$ and $b$ decays. For some operators, mainly those involving left-handed quark fields, the low energy constraints exclude a detectable LHC signal, whereas other operators are still allowed to have large enough coefficients to yield detectable NP signals at the LHC (see, e.g., Ref. [70]).

The experimental richness of higgs physics, that several production mechanisms and many decay channels can be probed, are to a large extent due to the particular values of the Yukawa couplings. The quark and lepton couplings, and $Y_{t}$ in particular, are important for higgs decays, as well as to determine the production cross sections from $g g$ fusion, higgs-strahlung, $t \bar{t}$ and $W Z$ fusion. The LHC has (almost) measured the $h \tau^{+} \tau^{-}$coupling, and will also determine $h \mu^{+} \mu^{-}$and $h b \bar{b}$, if they are near their SM values. Should the LHC or another future collider detect deviations from the SM branching ratios or observe flavor-non-diagonal higgs decays, that would of course be incredibly significant (for a recent discussion, see, e.g., Ref. [71]). 
Any new particle that couples to the quarks and/or leptons, potentially introduces new flavor violating parameters. For example, in low energy supersymmetry, which is the favorite NP scenario of a large part of our community, squark and slepton couplings may yield measurable effects in FCNC processes and $C P$ violation, give rise to detectable charged lepton flavor violation (CLFV), such as $\mu \rightarrow e \gamma$, etc. Observable $C P$ violation is then also possible in neutral currents and electric dipole moments, for which the SM predictions are below the near future experimental sensitivities. The supersymmetric flavor problems, that TeV-scale SUSY models with generic parameters are excluded by FCNC and $C P$ violation measurements, can be alleviated in several scenarios: (i) universal squark masses, when $\Delta \tilde{m}_{\tilde{Q}, \tilde{D}}^{2} \ll \tilde{m}^{2}$ (e.g., gauge mediation); (ii) alignment, when $\left(K_{L, R}^{d}\right) 12 \ll 1$ (e.g., horizontal symmetry); (iii) heavy squarks, when $\tilde{m} \gg 1 \mathrm{TeV}$ (e.g., split SUSY). All viable models incorporate some of these ingredients. Conversely, if SUSY is discovered, mapping out its flavor structure may help answer questions about even higher scales, e.g., the mechanism of SUSY breaking, how it is communicated to the MSSM, etc.

An important implication of flavor constraints for SUSY searches is that the LHC bounds are sensitive to the level of (non-)degeneracy assumed. Most SUSY searches assume that the first two generation squarks, $\tilde{u}_{L, R}, \tilde{d}_{L, R}, \tilde{s}_{L, R}, \tilde{c}_{L, R}$, are all degenerate, which increases signal cross sections. Relaxing this assumption consistent with flavor bounds, results in substantially weaker squark mass limits from the LHC Run 1, around the $500 \mathrm{GeV}$ scale [55]. Thus, there is a tight interplay between the flavor physics and LHC high- $p_{T}$ searches for new physics. If there is new physics at the TeV scale, its flavor structure must be highly non-generic to satisfy current bounds, and measuring small deviations from the SM in the flavor sector would give a lot of information complementary to ATLAS \& CMS. The higher the scale of new physics, the less severe the flavor constraints are. If NP is beyond the reach of the LHC, flavor physics experiments may still observe robust deviations from the SM, which would point to an upper bound on the next scale to probe.

\section{Final comments and ultimate sensitivity}

The main points I tried to convey through some examples were:

- $C P$ violation and FCNCs are sensitive probes of short-distance physics in the SM and for NP;

- Flavor physics probes energy scales $\gg 1 \mathrm{TeV}$, the sensitivity limited by statistics, not theory;

- For most FCNC processes NP/SM $\gtrsim 20 \%$ is still allowed, so there is plenty of room for NP;

- Of the several tensions between data and SM predictions, some may soon become definitive;

- Precision tests of SM will improve by $10^{1}-10^{4}$ in many channels (including CLFV);

- There are many interesting theory problems, relevant for improving experimental sensitivity;

- Future data will teach us more about physics at shorter distances, whether NP is seen or not, and could point to the next energy scale to explore.

With several new experiments starting (NA62, KOTO, Belle II, mu2e, COMET, etc.) and the upcoming upgrade of LHCb, the flood of new data will be fun and exciting (see Refs. [72, 73, 17] for reviews of planned flavor experiments and their sensitivities). It will allow new type of measurements, and more elaborate theoretical methods to be used/tested. The upcoming experiments 
also challenge theory, to improve predictions and to allow more measurements to probe short distance physics with robust discovery potential. Except for the few cleanest cases, improvements on both sides are needed to fully exploit the future data sets. I am optimistic, as order of magnitude increases in data always triggered new theory developments, too.

It is also interesting to try to estimate the largest flavor physics data sets which would be useful to increase sensitivity to new physics, without being limited by theory uncertainties. ${ }^{3}$ For charged lepton flavor violation, the SM predictions (from penguin and box diagrams with neutrinos) are (tens of) orders of magnitudes below any foreseeable experimental sensitivity, so if technology allows significant improvements, I think the justification is obvious (as it is for electric dipole moment searches). In quark flavor physics the situation is more complex. Amusingly, even in 2030, there will be theoretically clean $B$ decay modes in which (experimental bound) $/ \mathrm{SM} \gtrsim 10^{3}$, e.g., $B \rightarrow \tau^{+} \tau^{-}, B \rightarrow e^{+} e^{-}$, and probably some more. However, based on what is known today, some observables will become limited by theory (hadronic) uncertainties. Identifying how far NP sensitivity can be extended is interesting, at least in principle, so below is a list for which 50/ab Belle II and 50/fb LHCb data will not even come within an order of magnitude of the ultimately achievable sensitivities. Of course, on the relevant time scale lots of progress will take place (for estimates of future lattice QCD uncertainties, see, Ref. [74]) and new breakthroughs are also possible.

- Probably the theoretically cleanest observable in the quark sector is the determination of the CKM phase $\gamma$ from tree-level $B$ decays. Irreducible theory uncertainty only arises from higher order weak interaction [75]. So the main challenges are on the experimental side.

- The theory uncertainty for the semileptonic $C P$ asymmetries, $a_{\mathrm{SL}}^{d, s}$, discussed is Sec. 2 and in Fig 5, are also much below [76, 77] the expected 50/ab Belle II and 50/fb LHCb sensitivities.

- Another set of key observables are $B_{s, d} \rightarrow \mu \mu$ and $B \rightarrow \ell v$, where the nonperturbative theory inputs are only the decay constants, which will soon be known with $<1 \%$ uncertainties. In contrast, the expectation for the accuracy of $B_{d} \rightarrow \mu \mu$ with the full LHC data is $\mathscr{O}(20 \%)$.

- It is often stated that the determination of $\left|V_{u b}\right|$ is theory limited. This entirely depends on the measurements available. In principle, the theoretically cleanest $\left|V_{u b}\right|$ determination I know, which only uses isospin, would be from $\mathscr{B}\left(B_{u} \rightarrow \ell \bar{v}\right) / \mathscr{B}\left(B_{d} \rightarrow \mu^{+} \mu^{-}\right)$[78].

- I think that the SM prediction for $C P$ violation in $D^{0}-\bar{D}^{0}$ mixing is below the expected sensitivities on LHCb and Belle II. To establish this robustly, however, more theory work is needed (especially given the recent history of hints of $C P$ violation in $D$ decay).

- For $K^{+} \rightarrow \pi^{+} v \bar{v}$ and especially for $K_{L} \rightarrow \pi^{0} v \bar{v}$, the current plans for NA62 and KOTO will stop short of reaching the ultimate sensitivity to NP achievable in these decays.

Thus, I guess(timate) that $\sim 100$ times the currently envisioned 50/ab Belle II and 50/fb LHCb data sets would definitely allow for the sensitivity to short distance physics to improve. Whether any of these ultimate sensitivities can be achieved at a tera- $Z$ machine, an $e^{+} e^{-}$collider running on the $\Upsilon(4 S)$, or utilizing more of the LHC's or/and a future hadron collider's full luminosity, is something I hope we shall soon have even more compelling reasons to seriously explore.

\footnotetext{
${ }^{3}$ In measurements without SM backgrounds, such as setting bounds on $\mu \rightarrow e$ conversion or $\tau \rightarrow 3 \mu$ decay, the

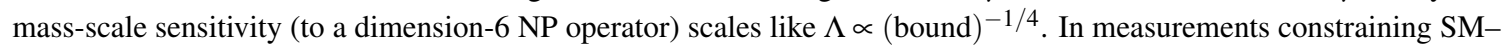

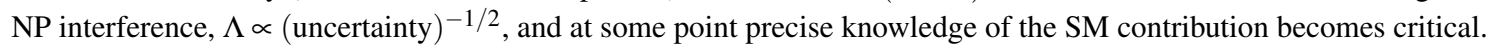


Acknowledgments I thank Marat Freytsis, Tim Gershon, Koji Ishiwata, Michele Papucci, Dean Robinson, Josh Ruderman, Filippo Sala, Karim Trabelsi, Phill Urquijo, and Mark Wise, for recent collaborations and/or discussions that shaped the views expressed in this talk. This work was supported in part by the Director, Office of Science, Office of High Energy Physics of the U.S. Department of Energy under contract DE-AC02-05CH11231.

\section{References}

[1] A. Abdesselam et al. [Belle Collaboration], arXiv:1603.06711.

[2] A. Abdesselam et al. [Belle Collaboration], arXiv:1604.04042.

[3] M. Aaboud et al. [ATLAS Collaboration], arXiv:1604.04263.

[4] R. Aaij et al. [LHCb Collaboration], arXiv:1605.09768.

[5] A. Höcker, H. Lacker, S. Laplace and F. Le Diberder, Eur. Phys. J. C 21, 225 (2001) [hep-ph/0104062]; J. Charles et al., Eur. Phys. J. C 41 (2005) 1 [hep-ph/0406184]; and updates at http://ckmfitter.in2p3.fr/.

[6] Z. Ligeti, arXiv:1502.01372.

[7] See the reviwes on the "Cabibbo-Kobayashi-Maskawa quark mixing matrix" and on " $C P$ violation in the quark sector" in: K. A. Olive et al. [Particle Data Group], Chin. Phys. C 38, 090001 (2014).

[8] K. Miyabayashi, talk at this conference, http://indico.cern.ch/event/325831/contributions/757780/.

[9] J. P. Lees et al. [BaBar Collaboration], Phys. Rev. Lett. 109, 101802 (2012) [arXiv:1205.5442].

[10] J. P. Lees et al. [BABAR Collaboration], Phys. Rev. D 88, 072012 (2013) [arXiv:1303.0571].

[11] M. Huschle et al. [Belle Collaboration], Phys. Rev. D 92, no. 7, 072014 (2015) [arXiv:1507.03233].

[12] R. Aaij et al. [LHCb Collaboration], Phys. Rev. Lett. 115, no. 11, 111803 (2015) Addendum: [Phys. Rev. Lett. 115, no. 15, 159901 (2015)] [arXiv:1506.08614].

[13] Heavy Flavor Averaging Group, Y. Amhis et al., arXiv:1412.7515; and updates at http://www.slac.stanford.edu/xorg/hfag/.

[14] J. A. Bailey et al. [MILC Collaboration], Phys. Rev. D 92, no. 3, 034506 (2015) [arXiv:1503.07237].

[15] H. Na et al. [HPQCD Collaboration], Phys. Rev. D 92, no. 5, 054510 (2015) [arXiv:1505.03925].

[16] S. Fajfer, J. F. Kamenik and I. Nisandzic, Phys. Rev. D 85, 094025 (2012) [arXiv:1203.2654].

[17] B. Golob, K. Trabelsi, P. Urquijo, BELLE2-NOTE-0021, https://belle2.cc.kek.jp/ twiki/pub/B2TiP/WebHome/belle2-note-0021.pdf.

[18] M. Freytsis, Z. Ligeti and J. T. Ruderman, Phys. Rev. D 92, no. 5, 054018 (2015) [arXiv:1506.08896].

[19] I. de Medeiros Varzielas and G. Hiller, JHEP 1506, 072 (2015) [arXiv:1503.01084].

[20] M. Freytsis, Z. Ligeti, J. Ruderman, to appear.

[21] Z. Ligeti and F. J. Tackmann, Phys. Rev. D 90, no. 3, 034021 (2014) [arXiv:1406.7013];

A. F. Falk, Z. Ligeti, M. Neubert and Y. Nir, Phys. Lett. B 326, 145 (1994) [hep-ph/9401226].

[22] B. Aubert et al. [BaBar Collaboration], Phys. Rev. D 79, 012002 (2009) [arXiv:0809.0828].

[23] W. Dungel et al. [Belle Collaboration], Phys. Rev. D 82, 112007 (2010) [arXiv:1010.5620].

[24] A. Greljo, G. Isidori and D. Marzocca, JHEP 1507, 142 (2015) [arXiv:1506.01705].

[25] E. C. F. S. Fortes and S. Nussinov, Phys. Rev. D 93, 014023 (2016) [arXiv:1508.04463].

[26] F. U. Bernlochner, Phys. Rev. D 92, no. 11, 115019 (2015) [arXiv:1509.06938]. 
[27] P. Hamer et al. [Belle Collaboration], Phys. Rev. D 93, no. 3, 032007 (2016) [arXiv:1509.06521].

[28] S. Descotes-Genon, J. Matias and J. Virto, Phys. Rev. D 88, 074002 (2013) [arXiv:1307.5683].

[29] W. Altmannshofer and D. M. Straub, Eur. Phys. J. C 75, no. 8, 382 (2015) [arXiv:1411.3161].

[30] R. Aaij et al. [LHCb Collaboration], JHEP 1602, 104 (2016) [arXiv:1512.04442].

[31] G. Lanfranchi, talk at this conference, http://indico.cern.ch/event/325831/contributions/757779/.

[32] S. Descotes-Genon, L. Hofer, J. Matias and J. Virto, JHEP 1412, 125 (2014) [arXiv:1407.8526].

[33] C. W. Bauer, D. Pirjol and I. W. Stewart, Phys. Rev. D 67, 071502 (2003) [hep-ph/0211069].

[34] M. Beneke and T. Feldmann, Nucl. Phys. B 685, 249 (2004) [hep-ph/0311335].

[35] J. Charles, A. Le Yaouanc, L. Oliver, O. Pene and J. C. Raynal, Phys. Rev. D 60, 014001 (1999) [hep-ph/9812358].

[36] S. Jäger and J. Martin Camalich, Phys. Rev. D 93, no. 1, 014028 (2016) [arXiv:1412.3183].

[37] M. Ciuchini, M. Fedele, E. Franco, S. Mishima, A. Paul, L. Silvestrini and M. Valli, arXiv:1512.07157.

[38] G. Hiller, Plenary talk at EPS 2015, https://indico.cern.ch/event/356420/contributions/1764018/.

[39] C. Bobeth, G. Hiller and D. van Dyk, Phys. Rev. D 87, no. 3, 034016 (2013) [arXiv:1212.2321].

[40] Z. Ligeti and F. J. Tackmann, Phys. Lett. B 653, 404 (2007) [arXiv:0707.1694]; K. S. M. Lee, Z. Ligeti, I. W. Stewart and F. J. Tackmann, Phys. Rev. D 75, 034016 (2007) [hep-ph/0612156].

[41] R. Aaij et al. [LHCb Collaboration], JHEP 1509, 179 (2015) [arXiv:1506.08777].

[42] A. Bharucha, D. M. Straub and R. Zwicky, arXiv:1503.05534.

[43] R. R. Horgan, Z. Liu, S. Meinel and M. Wingate, PoS LATTICE 2014, 372 (2015) [arXiv:1501.00367].

[44] V. Khachatryan et al. [CMS and LHCb Collaborations], Nature 522, 68 (2015) [arXiv:1411.4413].

[45] V. M. Abazov et al. [D0 Collaboration], Phys. Rev. D 84, 052007 (2011) [arXiv:1106.6308].

[46] Z. Ligeti, M. Papucci, G. Perez and J. Zupan, Phys. Rev. Lett. 105, 131601 (2010) [arXiv:1006.0432].

[47] F. U. Bernlochner, Z. Ligeti and S. Turczyk, Phys. Rev. D 90, no. 9, 094003 (2014) [arXiv:1408.2516].

[48] K. J. de Vries et al., Eur. Phys. J. C 75, no. 9, 422 (2015) [arXiv:1504.03260].

[49] M. Pospelov, talk at this conference, http://indico.cern.ch/event/325831/contributions/757807/.

[50] Z. Ligeti and F. Sala, arXiv:1602.08494.

[51] Z. Bai et al. [RBC and UKQCD Collaborations], Phys. Rev. Lett. 115, no. 21, 212001 (2015) [arXiv:1505.07863]; Erratum, arXiv:1603.03065.

[52] M. Czakon, P. Fiedler and A. Mitov, Phys. Rev. Lett. 115, no. 5, 052001 (2015) [arXiv:1411.3007].

[53] G. Isidori, J. F. Kamenik, Z. Ligeti and G. Perez, Phys. Lett. B 711, 46 (2012) [arXiv:1111.4987].

[54] O. Gedalia, J. F. Kamenik, Z. Ligeti and G. Perez, Phys. Lett. B 714, 55 (2012) [arXiv:1202.5038].

[55] R. Mahbubani, M. Papucci, G. Perez, J. T. Ruderman and A. Weiler, Phys. Rev. Lett. 110, no. 15, 151804 (2013) [arXiv:1212.3328].

[56] Y. Nir and N. Seiberg, Phys. Lett. B 309, 337 (1993) [hep-ph/9304307].

[57] R. Aaij et al. [LHCb Collaboration], Phys. Rev. Lett. 115, no. 16, 161802 (2015) [arXiv:1508.04094].

[58] M. Freytsis and Z. Ligeti, Phys. Rev. D 83, 115009 (2011) [arXiv:1012.5317]. 
[59] M. Freytsis, Z. Ligeti and J. Thaler, Phys. Rev. D 81, 034001 (2010) [arXiv:0911.5355].

[60] R. Godang et al. [CLEO Collaboration], Phys. Rev. Lett. 80, 3456 (1998) [hep-ex/9711010].

[61] Y. Grossman, A. L. Kagan and Z. Ligeti, Phys. Lett. B 538, 327 (2002) [hep-ph/0204212].

[62] M. Jung, Phys. Rev. D 86, 053008 (2012) [arXiv:1206.2050].

[63] P. Frings, U. Nierste and M. Wiebusch, Phys. Rev. Lett. 115, 061802 (2015) [arXiv:1503.00859].

[64] Z. Ligeti and D. J. Robinson, Phys. Rev. Lett. 115, no. 25, 251801 (2015) [arXiv:1507.06671].

[65] M. Jung, Phys. Lett. B 753, 187 (2016) [arXiv:1510.03423].

[66] J. Charles, S. Descotes-Genon, Z. Ligeti, S. Monteil, M. Papucci and K. Trabelsi, Phys. Rev. D 89, no. 3, 033016 (2014) [arXiv:1309.2293].

[67] A. F. Falk, Y. Grossman, Z. Ligeti and A. A. Petrov, Phys. Rev. D 65, 054034 (2002) [hep-ph/0110317]; A. F. Falk, Y. Grossman, Z. Ligeti, Y. Nir and A. A. Petrov, Phys. Rev. D 69, 114021 (2004) [hep-ph/0402204].

[68] K. Ishiwata, Z. Ligeti and M. B. Wise, JHEP 1510, 027 (2015) [arXiv:1506.03484].

[69] A. Meyer, talk at this conference, http://indico.cern.ch/event/325831/contributions/757774/.

[70] P. J. Fox, Z. Ligeti, M. Papucci, G. Perez and M. D. Schwartz, Phys. Rev. D 78, 054008 (2008) [arXiv:0704.1482].

[71] Y. Nir, CERN-2015-001, pp.123-156 [arXiv:1605.00433].

[72] Y. Sakai, talk at this conference, http://indico.cern.ch/event/325831/contributions/757784/.

[73] R. Aaij et al. [LHCb Collaboration], Eur. Phys. J. C 73, no. 4, 2373 (2013) [arXiv:1208.3355].

[74] J. N. Butler et al. [Quark Flavor Physics Working Group Collaboration], arXiv:1311.1076.

[75] A. F. Falk, private communications, unpublished (2001);

J. Brod and J. Zupan, JHEP 1401, 051 (2014) [arXiv:1308.5663].

[76] S. Laplace, Z. Ligeti, Y. Nir and G. Perez, Phys. Rev. D 65, 094040 (2002) [hep-ph/0202010].

[77] A. Lenz and U. Nierste, arXiv:1102.4274.

[78] B. Grinstein, Plenary talk at CKM 2006, http://ckm2006.hepl.phys.nagoya-u.ac.jp/slide/Plenary.html. 